Revue d'histoire de l'Amérique française

REVUE D.HISTOIRE DE L'AMÉRIQUE FRANÇAISE

\title{
Lionel Groulx, les indépendantistes de La Nation et le séparatisme (1936-1938)
}

\section{Robert Comeau}

Volume 26, numéro 1, juin 1972

URI : https://id.erudit.org/iderudit/303153ar

DOI : https://doi.org/10.7202/303153ar

Aller au sommaire du numéro

Éditeur(s)

Institut d'histoire de l'Amérique française

ISSN

0035-2357 (imprimé)

1492-1383 (numérique)

Découvrir la revue

Citer cet article

Comeau, R. (1972). Lionel Groulx, les indépendantistes de La Nation et le séparatisme (1936-1938). Revue d'histoire de l'Amérique française, 26(1), 83-102. https://doi.org/10.7202/303153ar d'utilisation que vous pouvez consulter en ligne.

https://apropos.erudit.org/fr/usagers/politique-dutilisation/ 


\title{
LIONEL GROULX, LES INDÉPENDANTISTES DE LA NATION ET LE SÉPARATISME $(1936-1938)^{*}$
}

\author{
ROBERT COMEAU \\ Département d'histoire \\ Université du Québec à Montréal \\ "Toute mystique est réactionnaire, \\ l'homme réactionnaire est mystique." **
}

La crise économique donna naissance à de multiples mouvements de contestation à caractère nationaliste au Québec. Ces mouvements, à travers la critique du régime Taschereau, s'en prenaient au libéralisme économique, à la démocratie parlementaire et à la "trahison des élites". Ils préconisaient une troisième voie entre le capitalisme libéral et le communisme, qu'ils combattaient violemment. Empruntant aux fascistes européens les idées corporatistes, ils ne remettaient pas en cause le système d'exploitation économique et la plupart d'entre eux, simples autonomistes, ne préconisaient pas la fin du régime fédératif. Quelques mouvements séparatistes se démarquent toutefois du courant autonomiste. Walter O'Leary en a brièvement fait l'historique :

Le premier des mouvements d'indépendance par le Québec est certes celui des Jeunesses Patriotes du Canada français, lequel est né spontanément, en pleine rue, soit au pied du monument Chénier en 1935. Il y a bien les Jeune-Canada avec André Laurendeau, Gérard Filion, etc., mais ce mouvement n'est pas unanimement pour l'indépendance du Québec. Les Jeunesses Patriotes comprenaient des indépendantistes connus tels que W. P. O'Leary, Jean-Paul Robillard, Michel Chartrand, Philippe Vaillancourt, Dostaler O'Leary, JeanLouis Gagnon, Jean Séguin, l'abbé Camille [sic] Brouillard, François Hertel, etc. Dès 1937, elles sont vite noyautées, puis divisées artificiellement en gauche et en droite. /.../ Nous pouvons citer aussi les Jeunes Laurentiens en 1937.1

* Sur ce groupe indépendantiste, voir Robert Comeau, Les indépendantistes québécois, 1936-1938. Thèse de M. A. (histoire), Université de Montréal, 1971. $212 \mathrm{p}$.

** Wilhelm Reich, La psychologie de masse du fascisme (Payot, Collection Science de l'homme, Paris, 1972), 45.

1 Walter O'Leary, "Petite histoire de l'indépendance du Québec", texte publié par le Centre d'étude de la Table Ronde du Québec libre, s. 1. (avril 1961).

RHAF, vol. 26, no 1 (juin 1972) 
Signalons également la revue Vivre à laquelle collaboreront deux futurs rédacteurs de La Nation, Pierre Chaloult et Jean-Louis Gagnon. Cette revue antidémocrate, violemment antibritannique, s'afficha séparatiste dès 1934. Le journal L'Indépendance, publié par les Jeunesses Patriotes, ne fut édité qu'à un seul numéro. C'est surtout La Nation et L'Unité Nationale qui présentèrent, à l'intérieur de l'idéologie nationaliste traditionnelle, l'option séparatiste. "En peu de temps ces deux publications se sont pour ainsi dire trouvées au centre d'une véritable tempête $/ . . . /$ qui a fait naître beaucoup d'animosité de la part des milieux officiels ou tout simplement conformistes." 2

Les Jeunesses Patriotes, quelques années avant la fondation de La Nation, s'étaient donné pour but "d'assurer une vaste fusion de tous les petits mouvements épars". ${ }^{3}$ A l'été 1935 , les quatre fondateurs de La Nation - Paul Bouchard, Albert Pelletier, Roger Vézina et Pierre Letarte - se réunirent en vue de publier un journal séparatiste au début de l'année 1936; ils avaient le même but: regrouper tous les éléments nationalistes à l'intérieur d'un vaste mouvement autonomiste. Ces membres propriétaires et fondateurs de La Nation étaient tous avocats, exmilitants libéraux pour la plupart, partageant la même sympathie pour Paul Gouin. Paul Bouchard était vraiment l'âme dirigeante de $L a$ Nation: il en assurait la direction et l'orientation idéologique. Ce leader autoritaire a toujours voulu voir incarnée dans un parti politique sa théorie nationaliste. Ayant échoué dans ses tentatives électorales à la tête de partis autonomistes, il placera son talent d'organisateur au service de l'Union Nationale, après avoir vivement combattu Maurice Duplessis.

Ce mouvement séparatiste nous apparaît "en continuité directe avec le mouvement nationaliste traditionnel", comme l'a noté Marcel Rioux. ${ }^{4}$ Le mouvement de Paul Bouchard, contrairement à ce qu'a pu affirmer Léon Dion, ${ }^{5}$ ne peut se comprendre en dehors de la perspective de l'abbé Groulx, qui exerça au cours des années trente une influence extraordinaire sur tous les mouvements nationalistes. C'est dans les écrits et les discours de leur maître à penser qu'ils disent trouver les justifications de leurs revendications indépendantistes. Carmel Brouillard, des Jeunesses Patriotes, s'est expliqué là-dessus:

\footnotetext{
257.

${ }^{2}$ Hector Grenon, Chroniques vécues, 1930-1940 (Ed. du Jour, 1967),

3 Ibid., 91.

4 Marcel Rioux, "Sur l'évolution des idéologies", Revue de Sociologie de l'Institut libre de Bruxelles, I (1968) : 110.

5 Léon Dion, "Le nationalisme pessimiste", Cité libre, 18 (nov. 1957) : 9.
} 
Que le théoricien de l'Etat français en Amérique répudie le séparatisme, il a ses raisons que nous comprenons et respectons. Mais rien n'empêchera ses livres et ses conférences d'affirmer ce qu'ils affirment. /.../ Ces pages seules suffisent à démontrer clairement notre destinée autonomiste et séparatiste. ${ }^{6}$

Cependant, avant d'aborder les rapports qu'ont entretenus avec l'abbé Groulx les séparatistes de La Nation de Québec, disons quelques mots de leur activité et de leur évolution politiques. Ces nationalistes petits-bourgeois qui joignent les rangs de la coalition antilibérale en dénonçant la dictature des monopoles, mais en rejetant avec autant d'énergie la lutte des classes et les tentatives d'organisation autonome de la classe ouvrière, présentent l'indépendance comme l'instrument de la libération de toute la nation. Ils participent activement à la campagne électorale contre Adélard Godbout; Paul Bouchard parle même de "séparatisme" à la radio, sous les auspices de "l'Union Nationale Duplessis-Gouin". Par la suite, ils seront plus critiques face à l'U.N. A quelques jours du scrutin, ils préconisent l'abstention, après avoir accordé leur appui aux candidats de l'U.N., comme l'avaient fait les Jeunesses Patriotes. Ils militent pour l'unité des nationalistes après la victoire électorale de Maurice Duplessis (17 août 1936) qui les a déçus rapidement. Ils mettent sur pied un comité de liaison des divers mouvements de jeunes nationalistes; à la tête de ce comité autonomiste on retrouve Philippe Ferland des Jeunesses Nationalistes, Walter O'Leary, président des Jeunesses Patriotes, Paul Dumas et Dostaler O'Leary des Jeune-Canada. Le groupe collabore aussi avec l'Union Nationale Ouvrière, mouvement d'extrême-droite, violemment anticommuniste qui voulait encadrer la population ouvrière. Paul Bouchard, déçu par Duplessis qui, au moment même de former son cabinet, a mis au rancart les éléments les plus radicaux, propose, dès décembre 1936, la création d'un grand parti autonomiste provincial. Le 25 mars 1937, La Nation devient l'organe d'un nouveau parti fédéral, le Parti Autonomiste, après s'être affichée "l'organe du mouvement séparatiste". La Nation mène une campagne pour gagner les nationalistes prestigieux de Québec ( $\mathrm{P}$. Hamel, O. Drouin, R. Chaloult, A. Marcoux, E. Ouellet) à l'idée de lancer un parti véritablement nationaliste qui pourrait remplacer l'Union Nationale. Après la fondation du Parti National par Philippe Hamel, Paul Bouchard invite, le 24 juin 1937, les anciens partisans montréalais de Paul Gouin

${ }^{6}$ Carmel Brouillard, Le séparatisme ne doit pas mourir, brochure (Editions des Jeunesses Patriotes, 1939). 
à se rallier à ce parti québécois. Les membres de la défunte Action Libérale Nationale ne réussiront pas à s'entendre avec les dirigeants du Parti National. L'année suivante, lorsque Paul Gouin réapparaît sur la scène politique pour susciter un regroupement de toutes les forces oppositionnistes contre le régime Duplessis, La Nation organise un grand congrès à Sorel pour ressusciter l'A.L.N., espérant que les dirigeants du Parti National accepteront d'y participer. Oscar Drouin, qui avait été chargé de l'organisation générale de l'A.L.N., est particulièrement réticent à ce ralliement autour de Paul Gouin qui a si lamentablement été écarté par Maurice Duplessis.

A la suite de ces échecs, Paul Bouchard lance, sur la scène fédérale, un nouveau parti, le 20 novembre 1938, le Parti Nationaliste dont le slogan réclame des "provinces autonomes dans un Canada libre". La Nation défend maintenant ce nouveau manifeste électoral qui offre, au dire de ses rédacteurs, "l'avantage de concilier les exigences du nationalisme canadien-français avec celles du patriotisme canadien". ${ }^{7}$ Les séparatistes de 19361937 en sont venus à modifier considérablement leur ligne politique initiale. On peut distinguer quatre options. Ils sont d'abord unanimes à déclarer le séparatisme nécessaire et possible (de février 1936 à mars 1937) ; ils préconisent ensuite un "réaménagement de la structure fédérale en une fédération d'états" (de mars 1937 à décembre 1937). Dans un troisième temps, ils proclament la nécessité d'acquérir de nouveaux pouvoirs afin d'augmenter la marge d'autonomie provinciale jugée insuffisante (de décembre 1937 à octobre 1938). Enfin, s'il est impossible d'obtenir de nouveaux pouvoirs, que l'on maintienne le statu quo, l'important étant de mettre un frein au processus de centralisation (octobre 1938 à août 1939).

Nous ne pouvons expliquer longuement ici comment ces séparatistes intransigeants en sont venus à promouvoir le maintien du statu quo, à mesure que les tentatives centralisatrices du gouvernement fédéral se faisaient plus pressantes et que la question de la participation au deuxième conflit mondial posait en priorité les rapports du Canada avec l'Angleterre. Le rôle qu'ils entendaient jouer dans la politique active et l'importance qu'ils accordaient à l'action électorale, contrairement aux autres intellectuels séparatistes des années trente, les ont obligés à amenuiser le caractère radical de leurs propositions en faveur du changement constitutionnel. Pour réaliser leur premier objectif,

${ }^{7}$ La Nation, 24 novembre 1938. 
l'unité des nationalistes, ils ne pouvaient accepter la marginalité à laquelle le radicalisme de leur option les condamnait. Pour expliquer le ralliement de ces séparatistes à l'autonomisme, il ne faudrait peut-être pas négliger, en plus des circonstances politiques, l'influence de l'abbé Groulx.

L'abbé Lionel Groulx est certainement l'homme que les séparatistes des années 1930 tenaient en plus haute estime: on n'a que des louanges pour "le maître de l'heure, le seul homme depuis Papineau qui a su donner une orientation à la nation". ${ }^{8}$ L'abbé Groulx est véritablement l'objet d'un culte. Paul Bouchard, dans un éditorial sur le centenaire des Patriotes de 1837, dira du "messie providentiel de notre délivrance future" ${ }^{\text {: }}$

Viendra celui qui devait venir, viendra celui qui reprendra l'idéal des Patriotes, qui prêchera la reconstruction de la Nouvelle-France. Et logiquement, c'est lui qui réhabilitera Papineau. Grâce à lui, il redeviendra un grand vivant. Par l'abbé Groulx, notre peuple réapprendra que Papineau fut "notre premier chef national" et qu'il y a lieu de se demander si aujourd'hui sans le tribun prestigieux, il existerait encore un Canada français ? ${ }^{10}$

A plusieurs reprises, on lui attribue la "paternité du regain nationaliste". ${ }^{11}$ Ainsi André Laurendeau, à ce moment-là président des Jeune-Canada, reconnaissait la fascination qu'exerçait alors l'abbé Groulx:

En premier lieu il faut reconnaître le triomphe de l'abbé Groulx. /.../ Si l'ouragan qui aurait pu être socialiste fut national au fond ce fut à cause de ce petit prêtre qui jamais ne fit de politique. /.../ Son œuvre alimenta et dirigea toute une presse indépendante: par l'intérieur, par la seule force de son rayonnement (mais quelle force vaut le rayonnement ?) elle suscita ces jeunesses nationalistes, Jeune-Canada, Jeunesses Patriotes, La Nation, Jeunesses de l'A.L.N. dont la crânerie et la vigoureuse sincérité furent un élément de la victoire [électorale de M. Duplessis en 1936]. ${ }^{12}$

On se porte à sa défense lorsqu'on le croit menacé par le gouvernement Duplessis. ${ }^{13}$ On rappelle souvent la "combine Tasche-

\footnotetext{
${ }^{8}$ P. Bouchard, La Nation, 7 janvier 1937.

9 Ibid., 1 er juillet 1937.

10 Ibid., 7 janvier 1937.

11 Voir en particulier, P. Talbot, "L'influence de l'abbé Groulx", La Nation, 14 mai 1936.

12 A. Laurendeau, "M. Duplessis met de l'eau dans son vin", La Nation, $1^{\text {er }}$ octobre 1936. Il s'agit de l'unique article de A. L. signé dans La Nation.

13 Paul Bouchard se dit prêt à faire intervenir Mgr Gauthier et le cardinal Villeneuve pour rappeler M. Duplessis à la raison. Voir "La tête de l'abbé Groulx serait la rançon du sauvetage de l'Université de Montréal", La Nation, 30 septembre 1937.
} 
reau-Beaubien-Dandurand" qui selon La Nation l'aurait obligé à renoncer à $L$ 'Action française. Le sénateur Beaubien avait exigé de Groulx l'engagement écrit d'enseigner l'histoire "dans la théorie conforme au pacte confédératif"; ce dernier avait refusé mais avait dû renoncer à L'Action française.

La Nation le proposera au poste de surintendant de l'Instruction publique. Le journal antinationaliste Le Jour ira jusqu'à faire état d'un "projet élaboré dans les conciliabules secrets du nouveau Parti National à l'effet que ce parti de Québec, appuyé par nombre de jeunes gens, manœuvre actuellement pour décider l'abbé Groulx, apôtre du séparatisme [sic], à accepter en principe de devenir premier ministre des Canadiens français". ${ }^{14}$

Ses livres et ses discours sont toujours chaleureusement commentés par les rédacteurs de La Nation, ce qui fera dire au libéral Edmond Turcotte qu'ils “ont été hypnotisés par l'abbé Groulx". ${ }^{15}$ Leurs réactions à la suite du célèbre discours du 29 juin 1937 au deuxième Congrès de la Langue française, où Groulx avait lancé "Notre Etat français, nous l'aurons", ${ }^{16}$ nous le laisse croire :

... l'abbé Groulx marque désormais la fin d'une époque et le commencement d'une autre; son discours, ceux qui montent l'apprendront par cœur, nous le ferons lire dans tous les foyers canadiens, ce sera notre Marseillaise à nous, notre chant du Départ vers la liberté et les plus hautes réalisations. ${ }^{17}$

\section{Groulx, caution des séparatistes?}

Les séparatistes de Québec veulent être cautionnés par le “maître de l'heure", parce qu'il se dit le porte-parole de l'exaspération de la jeune génération et à cause de son prestige. Il était bien

\footnotetext{
14 "M. Lionel Groulx, premier ministre", Le Jour, 16 septembre 1937.

15 P. Bouchard, La Nation, 29 février 1936.

16 On ne cessa de s'interroger sur le sens de cette déclaration. Le journal L'Autorité, sous la manchette "Une guerre civile dans le Québec", écrira : "On crut bien à un moment donné que certains extrémistes n'hésiteraient pas à pousser la jeunesse jusqu'à la guerre civile, pour atteindre des fins illusoires que seuls pouvaient rêver des habitants de la lune opaline", 3 juillet 1937. Le Journal de Québec commentera longuement en précisant qu'en fait "M. l'abbé Groulx n'est pas allé si loin que de vouloir nous détacher de la Confédération", $1^{\mathrm{er}}$ juillet 1937. L'Unité de Montréal cite de larges extraits en signalant que Groulx a montré "qu'il n'était pas forcément nécessaire de nous séparer du Dominion du Canada, à la condition bien évidente de faire du Dominion une véritable Confédération...", 8 juillet 1937.
}

17 P. Bouchard, La Nation, $1^{\text {er }}$ juillet 1937. 
normal que les jeunes radicaux essaient d'associer l'abbé Groulx à leur entreprise. Dans une lettre adressée à Lionel Groulx, le 22 janvier 1936, Paul Bouchard, après avoir annoncé la création de l'hebdomadaire La Nation et précisé son orientation séparatiste, lui demande un article:

... nous voulons consacrer les meilleurs de nos articles à la propagande séparatiste, à la formation d'un état libre français en Amérique, à la construction d'une nation nouvelle, libre et forte. Nous avons conçu un programme des plus élaborés et nous l'exposerons sans fléchir. /.../ Cela nous aiderait beaucoup et contribuerait mieux que nos écrits à répandre vos [sic] doctrines. ${ }^{18}$

L'abbé Groulx accepte que l'on reproduise dans La Nation une lettre adressée à Pierre Chaloult en date du 8 novembre 1935 dans laquelle il précise ses positions. Il avoue que, dès 1904, il proposait "cet idéal politique et national" d'un Canada français indépendant, tout en se défendant de n'avoir "jamais préconisé une rupture brusquée”. En expliquant ses prises de position séparatistes de 1922, il maintient que la Confédération va "se rompre d'elle-même" et il refuse de prendre l'initiative de "provoquer ou précipiter la séparation". Pour lui, la tâche n'est pas tant de changer les institutions que les hommes. C'est pourquoi, avoue-t-il, il importe d'abord de "réformer l'âme avant d'exiger l'action". Même s'il ne cache pas sa sympathie pour le groupe de séparatistes, ${ }^{19}$ nous pouvons affirmer que Groulx tenait à maintenir une certaine distance face au groupe des jeunes radicaux séparatistes de Québec. Même si, à long terme, il croit avec eux que nous devons tendre vers l'idéal d'un Canada français séparé, dans l'immédiat il tient à distinguer deux programmes d'action. Le sien, c'est un projet de "renaissance d'abord spirituelle". Puisque les Canadiens français sont responsables de ce qui leur arrive, il faut d'abord changer ces individus par divers moyens dont l'éducation nationale, à laquelle l'abbé Groulx attribue beaucoup de vertu. Fédéraliste, il attend ces Canadiens français réformés, “cent pour cent”, qui "replaceront à Ottawa et à Québec notre vie politique sur sa base normale", c'est-à-dire à l'intérieur de l'union fédérale. Groulx croit

18 Lettre de P. Bouchard à L. Groulx, le 22 janvier 1936. Correspondance conservée à la Fondation Lionel-Groulx et à l'Institut d'histoire de l'Amérique française.

19 "Je vous confesse que cet autre programme offre quelque chose de séduisant. Et il $\mathrm{y}$ a en tout cela une grande part de vérité. En tout cas, je ne me sens pas le goût de le désapprouver." Extrait de la lettre de L. Groulx à P. Chaloult du 8 novembre 1935, reproduite dans La Nation du 22 février 1936. 
que ces nationalistes pourront préparer un autre avenir en créant dans la province de Québec "tous les organismes d'un Etat viable". Il reconnaît que son programme "ne rencontre pas l'assentiment d'esprits fort judicieux de la jeune génération" pour qui la solution est prioritairement politique. Paul Bouchard dira lui-même vouloir traduire la doctrine de l'abbé Groulx sur le plan politique. ${ }^{20}$

L'abbé Groulx, tout en souhaitant l'indépendance, entretenait l'espoir que la Confédération puisse devenir un cadre acceptable alors que les rédacteurs de La Nation affirmaient en 1936 que l'indépendance était absolument nécessaire. Groulx allait beaucoup moins loin :

Proposons-leur la création d'un Canada français indépendant. Une lutte persévérante autour de cet objectif rétablira rapidement notre vie sur son fondement rationnel et elle redressera notre situation politique à Ottawa et à Québec. Et alors, ou la Confédération sera devenue un cadre acceptable; ou ce cadre nous l'aurons fait éclater par la vigueur même de nos aspirations. ${ }^{21}$

Il affirme même explicitement n'être pas prêt à se jeter dans l'aventure séparatiste qui n'aboutirait pour nous qu'à un "changement de maîtres", bien qu'il n'écarte pas totalement le programme séparatiste, incertain qu'un redressement soit encore possible dans le cadre de la Confédération. Les séparatistes de La Nation restent convaincus que l'abbé Groulx est foncièrement séparatiste, mais qu'il ne peut le déclarer:

Et en définitive, je dis à tous les crétins et à tous les intéressés qui s'empressent de clamer trop vite que l'abbé Groulx n'est pas séparatiste: Lisez soigneusement les textes de Groulx et vous verrez que la conclusion logique, c'est la séparation, que Groulx démolit admirablement l'argument sentimental des minorités françaises éparses dans le Dominion, que Groulx détruit tous les bobards qui forment l'idéal confédératif pour se contenter de nous donner une orientation. Si Groulx conseillait carrément alors ce serait une directive politique, ce que son caractère ecclésiastique et les ordres de ses supérieurs ne lui permettent pas. ${ }^{22}$

20 P. Bouchard, "L'abbé Groulx et le séparatisme", La Nation, 8 octobre 1936. Voir également M. Hamel, "Notre maître l'abbé Groulx", La Nation, 5 mai 1937 et le numéro spécial de La Nation consacré à Lionel Groulx le 2 septembre 1937.

21 Lettre de L. Groulx à Pierre Chaloult, le 8 octobre 1935.

22 P. Bouchard, "Séparatisme. L'abbé Groulx et le séparatisme", La Nation, 8 octobre 1936 . 
De même, Marcel Hamel, qui avait invité le "maître" à apporter son appui moral, tenait à lui rappeler que les idées de La Nation étaient les siennes: "Je fais partie de la rédaction d'un journal qui prône vos idées, qui les veut divulguer." 23 Dans une autre lettre où il lui demandait de rédiger un article pour La Nation, il ajoute:

Il me semble qu'un article de ce genre ne vous compromettrait aucunement vis-à-vis des autorités mitrées ou autres. Et pour nous, je ne vous le cache pas, ce serait une approbation indirecte de notre campagne /.../ Le maître reniera-t-il ses disciples? Sans votre support, il n'y a plus rien à entreprendre; le sol nous échappe sous le pied et nous dégringolerons comme les autres mouvements d'action française.

Dans l'espoir que vous vous rendrez à notre supplique, nous demeurons, de la Nation, vos fils spirituels et enthousiastes. ${ }^{24}$

L'abbé Groulx ne refusera pas son appui; il accepta de donner une conférence dont les fonds devaient aller à La Nation, mais qui fut annulée à la demande des séparatistes. Marcel Hamel s'expliquait ainsi à l'abbé Groulx:

Un cercle fondé à la dernière minute vous invite à venir prononcer une conférence avec des prix d'admission. Tout de suite ces dames s'enquièrent où vont les recettes; elles ont tôt fait de découvrir l'anguille sous roche. Vous voyez d'ici le scandale formidable. M. l'abbé Groulx, dira-t-on, qui s'est déclaré formellement non-séparatiste et qui encourage La Nation! J'avais donc raison d'une façon, quoique vous me blâmiez dans votre lettre à Mme Lacroix, d'entretenir des craintes sur cette intervention très amicale de votre part, mais qui pourrait, en définitive, vous causer nombre d'ennuis. C'est donc décidé: nous ne recourrons pas à votre obligeance pour sauver La Nation; Vous, avant n'importe qui et n'importe quoi !

Alors, M. l'abbé, il ne me reste plus qu'à vous remercier du plus profond du cœur pour ce que vous auriez fait pour nous! 25

Les séparatistes échangent une correspondance avec Groulx pour lui demander un appui moral, l'inviter à prononcer des conférences. ${ }^{26}$ On l'informe des conflits à l'intérieur des chapelles

23 Lettre de Marcel Hamel à Lionel Groulx, le 2 mai 1936. Correspondance conservée à la Fondation Lionel-Groulx et à l'Institut d'histoire de l'Amérique française.

24 Ibid., 12 août 1937.

25 Lettre de Marcel Hamel à Lionel Groulx, le 17 janvier 1938.

26 Voir lettre de P. Bouchard à L. Groulx du 2 juillet 1937. 
nationalistes. Par exemple, le 20 octobre 1937, le directeur de La Nation fait état à l'abbé Groulx des difficultés que lui occasionne le docteur Philippe Hamel en fondant son journal L'Ere Nouvelle: "Le Dr Hamel a fait le calcul qu'en publiant un journal, il parviendrait sans doute à faire tomber La Province [journal de Paul Gouin] qu'il trouve plate, et La Nation, qui est trop nationaliste à son gré." ${ }^{27}$ Cette correspondance fait également état des nombreuses querelles de personnalité opposant par exemple Paul Bouchard à Dostaler O'Leary, Jean-Louis Gagnon et Pierre Chaloult. On lui demande parfois de servir de conciliateur. ${ }^{28}$ Il est également informé des difficultés financières de La Nation. On lui demande conseil pour obtenir des fonds, ${ }^{29}$ et même les droits exclusifs sur l'édition de sa conférence au Congrès de la Langue française tenu à Québec le 29 juin $1937 .{ }^{30}$

Maintenant que nous avons vu comment les indépendantistes de Québec ont tenté de s'annexer l'abbé Groulx en mettant en sourdine leurs divergences politiques, analysons plus à fond l'option politique de ces disciples plus audacieux.

\section{Des disciples plus audacieux}

Dès le premier mois de la fondation de La Nation, le directeur précisait en quoi la position du groupe de Québec allait plus loin que celle de l'abbé Groulx. Pour les séparatistes de 1936, contrairement à ceux de 1922, l'attente passive du jour de l'indépendance devait faire place à la recherche de la rupture:

Le séparatisme de 1922 était l'idée d'une élite de patriotes qui voulait prouver que la sécession du Québec de la Confédération canadienne n'était pas une utopie; qu'elle pouvait survenir brusquement au milieu du siècle et nous prendre par surprise, et qu'à tout le moins il était philosophiquement permis d'y songer.

Pour nous, la nécessité impérieuse et l'urgence de la sécession ne laissent aucun doute $/ \ldots /$ parce que la société politique dont nous faisons partie est bâtarde et hybride, nous sommes maintenant dans un état d'infériorité qui entrave le libre épanouissement de nos facultés nationales. ${ }^{31}$

27 Lettre de P. Bouchard à L. Groulx du 20 octobre 1937.

28 Voir la correspondance de Paul Bouchard à Lionel Groulx; en particulier la lettre du 2 novembre 1937.

29 Lettre de P. Bouchard à L. Groulx du 9 mai 1936.

30 Lettre de P. Bouchard à L. Groulx du 2 juillet 1937.

31 Paul Bouchard, La Nation, 15 février 1936. 
A ceux qui refusent de militer activement sous prétexte que la Confédération doit prendre fin d'elle-même, il répond encore que "rien n'est moins prouvé":
$/ . . /$ car c'est mon opinion que ne périssent des institutions humaines que celles qu'on laisse tomber. Or la majorité anglo-saxonne du pays bénéficie de la Confédération et ne tient pas du tout à la laisser tomber. Elle s'y résignera devant le fait accompli, mais ne viendra pas nous offrir d'elle-même la séparation. /.../ La Confédération ne prendra fin que si nous voulons et que si nous agissons et prenons les moyens pour y mettre fin. ${ }^{32}$

Ceux qui se prétendent les véritables disciples de Groulx en 1936 et 1937 s'en prennent à plusieurs reprises à 'l'utopie d'un Etat francais dans la Confédération", ${ }^{33}$ ce que semble pourtant défendre l'historien montréalais. Dostaler O'Leary des Jeunesses Patriotes définissait clairement les deux options, rejetant celle de Groulx:

Les partisans d'une politique pro-canadienne-française se divisent donc encore en deux groupes: ceux qui croient à la possibilité d'un Etat français dans la Confédération et ceux qui ne croient cette réalisation possible, qu'en dehors des cadres du Canada actuel. /.../ La réalisation d'un Etat français dans la Confédération est une utopie, j'essaye de le démontrer au cours de ce bouquin. ${ }^{34}$

Les séparatistes se veulent dans la ligne de l'abbé Groulx. Ils veulent traduire sa doctrine nationale de l'Etat français en un programme d'action politique, séparatiste et corporatiste:

Sur la mystique essentielle de l'Etat français (la "doctrine de l'abbé Groulx") établissons des mystiques complémentaires. /.../ La doctrine séparatiste complète la doctrine nationale émise par l'abbé Groulx de mystiques politiques et économiques indispensables à un peuple moderne. C'est un pas en avant. Groulx nous a montré que la Confédération n'est pas viable. Nous en demandons la sécession dans l'avenir le plus immédiat et montrons ce qu'il faut construire. ${ }^{35}$

Même s'ils ne sont pas d'accord avec certaines déclarations politiques de Groulx, ils l'excusent presque de ne pouvoir être séparatiste: "Préparons-nous à devenir indépendant. Cette idée

32 Paul Bouchard, La Nation, 24 décembre 1936. 1936.

33 Voir par exemple l'article de P. Bouchard, La Nation, 17 décembre

34 Dostaler O'Leary, Séparatisme, doctrine constructive (Les éditions des Jeunesses Patriotes, Montréal, 1937), 17.

35 P. Bouchard, La Nation, 8 octobre 1936. 
maîtresse que nous avons reprise, nous à La Nation, avec plus d'audace - parce qu'il s'est écoulé plus d'années - Groulx en est et en restera devant les générations futures le véritable doctrinaire." ${ }^{36}$ De son côté, l'historien montréalais prend leur défense. Par exemple, au Congrès des instituteurs catholiques de Montréal, le 5 décembre 1936:

/.../ me permettez-vous de dire, en passant, à ceux qui me pressent de dénoncer le séparatisme, qu'avant de chercher querelle à cette école, avant de faire de l'ironie facile, puérile ou sénile au sujet de la Laurentie, je m'en prendrai aux éducateurs routiniers, aux chefs sans envergure, aux politiciens sans courage, aux anesthésistes de toute catégorie, qui ont rendu le mouvement séparatiste inévitable [sic]. En m'accrochant à la possibilité d'un Etat français dans la Confédération, il se peut que j'aie tort, que je sois même naïf. $3 \pi$

\section{Groulx et le séparatisme (1936-1938)}

Même si, dès 1936, les idées des séparatistes de Québec sont assimilées à celles de l'abbé Groulx par les fédéralistes, ${ }^{38}$ nous verrons que l'historien montréalais n'a jamais voulu s'afficher "séparatiste". Marcel Hamel affirme lui-même dans une lettre que l'abbé Groulx s'est déclaré "formellement non-séparatiste". ${ }^{39}$ Dans ses notes de cours qu'il a utilisées à l'Ecole de Vaudreuil, école de formation sociale destinée à la Jeunesse Indépendante Catholique, on peut lire:

Voilà, au moins quatre fois que, dans un an, je déclare n'être point séparatiste, et définis l'Etat français. /... / L'on ne peut plus concevoir d'Etat français que l'Etat séparatiste. Cependant nous vivons à côté des Etats-Unis: on devrait savoir ce que peut être un Etat autonome dans une fédération. 40

Cette mise au point avait été rendue nécessaire à la suite de la fracassante déclaration qu'il avait faite au deuxième Congrès 167-168.

${ }^{36}$ P. Chaloult, La Nation, 28 mai 1936.

37 Lionel Groulx, Directives (Saint-Hyacinthe, Editions Alerte, 1959),

38 Edmond Turcotte du journal Le Canada présente les séparatistes comme des disciples de l'abbé Groulx: "La chimère laurentienne de l'abbé Groulx a séduit quelques jeunes gens qui ont du courage à revendre et ne savent pas le dépenser" (cité dans La Nation du 29 février 1936).

39 Lettre de Marcel Hamel à Lionel Groulx du 17 janvier 1938.

40 Lionel Groulx, Leçons professées à l'Ecole de Vaudreuil, manuscrit non paginé, 15 août 1937. Partie II : L'Etat français, chapitre premier. Manuscrit déposé à la Fondation Lionel-Groulx et à l'Institut d'histoire de l'Amérique française. 
de la Langue française à Québec et qu'avaient reproduite tous les journaux:

Qu'on le veuille ou qu'on ne le veuille pas, notre Etat français, nous l'aurons; nous l'aurons jeune, fort, rayonnant et beau, foyer spirituel, pôle dynamique pour toute l'Amérique française. Nous aurons un pays français, un pays qui portera son âme dans son visage.

Groulx devait s'expliquer: son "Etat français" était-il l' "Etat libre" des séparatistes ? Devant les interprétations divergentes et, si l'on en croit Robert Rumilly, sous les pressions du cardinal Villeneuve, ${ }^{41}$ Groulx fit la mise au point suivante:

Dégageant à Québec aux "cours de vacances de pédagogie pour les institutrices", les leçons que comporte l'histoire du Canada et traitant des méthodes de la bien enseigner aux petites Canadiennes françaises, l'abbé Lionel Groulx a cru devoir faire une mise au point et bien préciser son attitude sur ce problème du séparatisme. "Je ne suis pas séparatiste, a-t-il déclaré. Quand je dis Etat français, je parle d'un Etat fédératif. Je reste dans la ligne de l'histoire. Nous ne sommes pas entrés dans la Confédération pour en sortir mais pour nous y épanouir." 42

Dans une autre leçon intitulée "Séparatisme et Etat français",43 il établit d'abord que "les Canadiens français ont le droit à la pleine réalisation de leur personnalité nationale", mais il s'interroge longuement quant à savoir si effectivement c'est bien le cadre confédératif qui rend impossible cette "pleine réalisation de la personnalité nationale". Il refuse de faire de la Confédération le bouc émissaire de tous les maux des Canadiens français. Il constate bien le "désordre économique et social, pauvreté généralisée et prolétarisation excessive _-", mais loin d'y voir les effets d'un système particulier d'exploitation capitaliste, Groulx s'en prend aux politiciens qui ont manqué de "sens national". L'idéaliste abbé Groulx a toujours cru que la classe dominante pouvait travailler à l'avantage de toutes les classes. Il rend donc responsables du désordre économique les dirigeants québécois, qui "avec une volonté mieux orientée"

41 Voir Robert Rumilly, Histoire de la Province de Québec (Fides, Montréal, 1967), XXXVI: 189-190. "Le cardinal Villeneuve avertit et freine l'abbé Groulx reprenant la thèse et presque les termes du Père Villeneuve. Et l'abbé Groulx doit se défendre publiquement d'entretenir l'idée séparatiste."

42 "Mise au point de l'abbé Groulx", Le Franc-Parleur, 12 août 1937.

43 Lionel Groulx, Leçons professées à l'Ecole de Vaudreuil, "Séparatisme et Etat français" (9 pages). Manuscrit déposé à la Fondation LionelGroulx et à l'Institut d'histoire de l'Amérique française. 
auraient pu "facilement concevoir un autre développement industriel de la province". Ce sont eux qui ont rendu le séparatisme inévitable sinon nécessaire. ${ }^{44}$ Par conséquent, il reconnaît "une grande part de légitimité dans l'attitude offensive des séparatistes". 45

$\mathrm{Si}$, à court terme, l'historien nationaliste a toujours évité de préconiser la séparation, il est évident qu'il a toujours cru nécessaire d'entretenir "l'idéal d'un Etat indépendant" et souhaité que se réalise un jour "l'Etat français indépendant". Celui qui a écrit que la "libre disposition de soi-même dans la pleine indépendance est l'état politique parfait" ${ }^{46}$ et qui n'a cessé d'interpréter l'histoire comme une longue marche de la nation canadienne-française vers la plus grande autonomie, l'indépendance, ne croyait pas à la possibilité d'une séparation immédiate. Il s'est interrogé longuement. Il distinguait la question de fait de la question de droit, en ces termes: "La Confédération durera-t-elle ? et Y devons-nous rester ?"

A la première question, il estime que les chances de durer sont bien minces. Son argument principal ne repose pas dans "l'absurdité géographique" et les multiples sectionnements du trop vaste territoire canadien, comme le soutient François Hertel, mais dans "la faiblesse du vouloir-vivre collectif" des Canadiens. Dans ses notes de cours, c'est le seul argument qu'il développe: le peu d'adhésion des citoyens au nationalisme pancanadien, causé par la structure politique et l'histoire. ${ }^{47} \mathrm{~A}$ ce sujet, il rappelle que "nos crises furent diviseuses plutôt qu'unificatrices". 48 Par conséquent, "rien n'oblige à croire à la pérennité de la Confédération". ${ }^{49}$

Abordant la question de droit (Devons-nous sortir de la Confédération ?), il invoque souvent trois arguments pour justifier son refus de préconiser l" "action séparatiste". Premièrement: "Un redressement est-il impossible, j'aimerais qu'on nous

44 Ibid.

$45 \mathrm{Ibid}$. On peut encore lire dans ses Notes de cours: "Le séparatisme - solution extrême qui deviendra nécessaire peut-être. Quand il faudra prendre le dernier risque."

46 Ibid.

47 Voir également sa conférence au Congrès des Jeunesses Patriotes, le 13 septembre 1936, reproduite dans Directives, 113-115.

48 Ibid.

49 L. Groulx, Leçons professées à l'Ecole de Vaudreuil, "Devoirs que nous impose notre nationalisme". Manuscrit non paginé conservé à la Fondation Lionel-Groulx et à l'Institut d'histoire de l'Amérique française. 
le démontrât de façon plus décisive." ${ }_{50}$ Groulx, qui se demande toujours si c'est la "faute des institutions ou la faute des hommes", mise tellement sur "l'éducation nationale" qu'il ne peut désespérer qu'on formera des élites qui ne trahiraient pas:

Je vous accorde qu'avec une race de Canadiens français, comme celle d'aujourd'hui, celle que nous connaissons, dégénérée politiquement, la Confédération ne peut nous être que mortelle. Serait-ce impossible avec une race nouvelle [sic] qui, au fétichisme du parti, aurait substitué un vigoureux attachement à son trésor culturel ? Je ne le crois pas. ${ }^{51}$

La deuxième question porte sur nos moyens: Sommes-nous capables d'assumer les risques de l'indépendance ? Compte tenu que nous ne sommes aucunement maîtres de notre vie économique, serions-nous un Etat viable ? Il veut pouvoir offrir la certitude d'un mieux-être: "Serions-nous en état de nous suffire? de procurer à nos nationaux leur bien humain, mieux que nous ne pouvons le faire en restant dans la Confédération? Ici encore, vous avez peut-être raison, mais j'aimerais que cela aussi, on nous le démontrât de façon irréfutable." "52 La troisième objection concerne le sort du Canada anglais dans l'hypothèse du séparatisme: "Notre sortie de la Confédération porterait-elle un tel coup à la Confédération qu'elle empêcherait nos associés

50 Ibid.

51 Ibid. Il est encore plus explicite à ce sujet dans Directives, 119. L'idéaliste abbé Groulx sous-estime les facteurs objectifs. Par exemple la conclusion de son article "Le problème économique" paru dans l'Action française (déc. 1921) et reproduit dans Directives (1959) est particulièrement significative: "Une cause existe, en vérité, de tous nos retards et de toutes nos faiblesses dans le domaine économique. Cette cause profonde, l'analyse que nous venons de faire a déjà permis de la pressentir: elle n'est pas en dehors de nous, elle est en nous (Directives, 38)." La même conclusion douloureuse vient clore le plus grand nombre des études dont se compose l'enquête: nous manquons de confiance en nous-mêmes. Ainsi au lieu d'engager ses lecteurs à une transformation des structures par une propagande matérialiste, c'est à la vaine tentative de changer les mentalités qu'il les convie, en les incitant à "vouloir" et à avoir confiance en eux-mêmes. Dans sa conférence, "L'économique et le national", présentée à la Jeune Chambre de Commerce de Montréal le 12 février 1936, il est encore plus explicite: "Notre mal, notre grand mal, il y a longtemps que je le dis: il n'est pas d'abord dans l'ordre politique, ni dans l'ordre économique; il est dans nos esprits. Car un peuple catholique et français qui ne laisse pas orienter sa vie par des idées directrices catholiques et françaises, mais par des vues superficielles et secondaires, ne peut être qu'un peuple qui bâtit toute sa destinée à l'envers. Et voilà pourquoi le désordre est total (Directives, 75-76)." nationalisme".

52 L. Groulx, Leçons professées... "Devoirs que nous impose notre 
d'aujourd'hui d'atteindre leur bien propre ?"53 Après avoir soulevé la question, il n'y répond pas ou plutôt la réduit à la question $\mathrm{du}$ sort des minorités françaises du Canada. Il démontre en quoi l'indépendance ne signifie pas l'isolement pas plus que l'abandon des minorités, attaquant assez vigoureusement les détracteurs du séparatisme. ${ }^{54}$ Dans sa causerie aux Jeunesses Patriotes il apporte une autre objection concernant la présence de la minorité anglophone du Québec; Groulx essaie de convaincre qu'une “politique nationale n'est pas nécessairement une politique d'injustice envers la minorité". Il ajoute: "J'estime, bien au contraire, que l'avènement d'un Etat français, à l'heure actuelle, dans le Québec, inaugurerait en même temps qu'une politique canadienne-française, une politique de suprême justice." Car il est bien clair pour lui que

la question est bien de savoir /.../ si une minorité d'exploiteurs, formant peut-être six pour cent de la population car tous les anglophones ne sont pas des exploiteurs - a le droit d'empêcher un peuple de 2,500,000 âmes, soit quatrevingt pour cent de la population de la province, de vivre librement et dignement. La question est même de savoir s'il est au pouvoir de ces six pour cent de perpétuer leur dictature. 55

Il croit cette fraction de la classe dominante assez lucide pour comprendre qu'il est dans son intérêt même "de se prêter à une solution de justice, par les voies pacifiques, plutôt que de laisser venir infailliblement une autre solution, s'il est vrai qu'un peuple n'accepte pas longtemps un régime inacceptable". ${ }^{56}$

D'une part, s'il n'incite pas à travailler au démembrement du pays, au nom du principe que “tant qu'il n'est pas avéré qu'un ordre politique est devenu injuste, et surtout tant qu'il n'est pas manifeste qu'il puisse être remplacé par un autre plus propre à pourvoir une nation du bien humain, un ordre politique vaut et est objet de justice sociale" ${ }^{57}$; d'autre part, il préconise la construction d'un Etat français:

Le devoir urgent, le devoir certain, où il n'y a pas de risque

de se tromper, ni de perdre son effort, c'est de travailler à

la création d'un Etat français dans le Québec - dans la

53 Ibid.

54 Ibid. Voir également Directives, 121.

55 L. Groulx, Directives, 112.

56 Ibid., 112 .

57 Citation du Père Lachance, tirée de Nationalisme et religion, 191; citée par L. Groulx, dans ses notes de cours, Ecole de Vaudreuil, août 1937. 
Confédération si possible - en dehors de la Confédération si impossible. C'est le moyen d'atteindre notre bien humain et peut-être même, s'il n'est pas trop tard, de redresser la Confédération. ${ }^{58}$

Quant aux moyens qu'il préconise pour forger "lentement, solidement les organes du futur Etat", ils relèvent plus de la mystique que de l'action politique:

Je ne demande donc pas de vaincre par la violence. Je vous demande de vaincre par les armes d'une jeunesse catholique et française: la probité, l'intelligence, le travail, la ténacité, l'audace généreuse. Je vous dirai même: ne précipitez rien. Soyez patients. Forgez lentement, solidement les organes du futur Etat. 59

Entre l'incitation à la lutte armée et le développement des vertus morales, Groulx ne propose rien de concret. Et la justification ultime de l'édification d'un Etat français et catholique repose sur la supériorité des valeurs spirituelles, car "travailler à la survivance, à la création d'un Canada français catholique, c'est travailler pour la grande réalité spirituelle en notre pays".60

\section{L'État français : une réalité présente ou un Etat à bâtir?}

Groulx a souvent parlé de l'Etat français du Québec, parfois comme d'une réalité présente, parfois comme d'un idéal à atteindre. Pour le groupe de radicaux séparatistes de Québec, les pouvoirs attribués au gouvernement québécois sont nettement insuffisants pour que l'on puisse parler d'un "Etat québécois". Les gouvernements provinciaux créés en 1867 ne sont à leur avis que "de grands conseils municipaux dépouillés soigneusement de tous les leviers de commande qui constituent un Etat"'.61 En décembre 1936, Paul Bouchard était encore plus explicite:

Et qu'est-ce que c'est que cet Etat qui ne possède pas au moindre degré ce qui fait la caractéristique essentielle de l'Etat: la souveraineté, sinon la souveraineté extérieure, du moins l'autonomie, la souveraineté intérieure; car un Etat peut être vassal d'un autre et posséder une souveraineté intérieure complète. Pour qu'il y ait un Etat selon les règles du droit international il faut au moins que la souveraineté intérieure soit complète. C'est pourquoi aucune des provinces

${ }^{58}$ L. Groulx, Leçons professées à l'Ecole de Vaudreuil.

59 L. Groulx, Directives, 123-124.

60 L. Groulx, Leçons professées ...

61 P. Bouchard, La Nation, 15 octobre 1936. 
de la Confédération canadienne ne peut être regardée comme un Etat. ${ }^{62}$

L'abbé Groulx croit, pour sa part, qu'en 1867 la province de Québec fut dotée d'un "Etat national" qui pour lui est synonyme d'Etat français, c'est-à-dire, selon sa définition, "apte à gouverner pour les nationaux, pour la majorité de la population canadienne-française". Groulx, comme tous les porte-parole de l'idéologie bourgeoise de l'époque, entretient l'idée selon laquelle l'Etat n'est pas inévitablement l'instrument d'une classe; il croit à la neutralité de l'Etat capitaliste qu'il situe au-dessus des classes et appelé à sauvegarder les intérêts de tout le peuple québécois. Il croit que les fondateurs du "B.N.A. Act" ont voulu créer un "Etat national" dans la province de Québec. Dans Directives, il ira jusqu'à écrire: “ce postulat de l'Etat français n'offre rien de nouveau. Il est dans la stricte ligne de notre histoire. Il a été progressivement réalisé à partir de 1774 . /.../ L'Etat français, dans la province de Québec est devenu depuis 1867 de droit positif, constitutionnel." 63 Il semble croire que le gouvernement du Québec avait les moyens, c'est-à-dire les ressources financières, les pouvoirs et la possibilité de les utiliser pour mettre sur pied une "politique nationale" qui aurait assuré l'émergence d'une bourgeoisie capitaliste autochtone. ${ }^{64} \mathrm{Si}$ une telle politique ne fut pas appliquée, la responsabilité doit en être attribuée aux politiciens.

D'autre part, Groulx parle aussi d'un Etat français à créer. Comment doit apparaître cet "Etat au service de la collectivité française" que l'on pourrait qualifier quant à nous, d'utopique ?

L'Etat français, il n'est d'ailleurs au pouvoir de personne de le créer artificiellement. Il existera lorsque nous en aurons créé les organes vitaux, et que ces organes, une âme puissante les pourra articuler. C'est pourquoi j'estime que ce n'est pas perdre son temps que de former la race de Canadiens français qui sera la première richesse du futur Etat ... ${ }^{65}$

Ce n'est pas nécessairement par l'octroi de nouveaux pouvoirs

62 P. Bouchard, La Nation, 17 décembre 1936.

63 L. Groulx, Directives, 108.

${ }^{64}$ Voir les chapitres 3 et 4 de Les indépendantistes québécois, 1936 1938. A travers les justifications que les séparatistes de La Nation ont apportées à l'appui de la thèse séparatiste (chap. 3), et à partir de l'analyse du programme économique qu'ils défendaient' (chap. 4), nous avons montré le caractère de classe de cette idéologie nationaliste "radicale"; nous avons tenté de démontrer en quoi ces intellectuels sont les représentants d'une fraction de la petite-bourgeoisie canadienne-française.

65 L. Groulx, Directives, 168. 
politiques qu'il doit être créé car, selon Groulx, le partage des pouvoirs de 1867 rendait possible cet "Etat français":

Et j'entends par là que l'une de nos conditions de vie consiste à créer, dans la province de Québec, ce qui devrait exister depuis 1867, ce qui est la conséquence logique, expresse, de la Confédération, l'aboutissant de notre histoire: un Etat français. 66

L'Etat français que préconisait l'abbé Groulx n'était pas l'Etat séparé que préconisaient les séparatistes. A quelques reprises, il a tenu à préciser que l'Etat français pouvait se réaliser à l'intérieur de la Confédération.

Roger Vézina, dans une analyse d'un discours de l'abbé Groulx, va jusqu'à dire que pour l'historien montréalais, "l'Etat français est beaucoup plus une manière d'être et de vivre qu'un simple organisme politique". ${ }^{67}$ L'abbé Groulx avait clairement dit: "Nul besoin, pour créer cet Etat, de changer un iota aux constitutions qui nous régissent." 68 Groulx accepte les postulats de ce que Maurice Séguin appelle "l'idéologie fédéraliste". ${ }^{69}$ Les indépendantistes, après avoir tant voulu s'annexer Groulx, deviennent autonomistes comme lui en 1938. Ils reconnaissent toutefois, à ce moment, ces différences:

On nous a souvent mis en opposition avec notre maître l'abbé Lionel Groulx. Nous allions en effet beaucoup plus loin que lui et persistions à croire que cela était nécessaire en théorie. Nous avons prêché le séparatisme, non comme réalisation brusque et immédiate, mais comme le lointain idéal où devait tendre et aspirer notre nationalité.70

Après avoir été séparatistes durant un peu plus de deux ans, ils adoptaient le 28 novembre 1938 la formule ambiguë de l'historien national: "Selon les désirs de notre maître l'abbé Groulx, nous sommes disposés à tenter encore une fois un loyal et final essai de la Confédération. /.../ Comme lui, nous voulons un Etat français dans le Québec et sommes prêts à l'édifier dans la Confédération si possible, hors de la Confédération si impos-

66 Ibid., 128-129.

67 R. Vézina, La Nation, 5 mai 1938.

68 Discours au Congrès des Jeunesses Patriotes, reproduit dans Directives, 109.

69 Maurice Séguin, L'Idée d'indépendance au Québec, genèse et historique (Les Editions Boréal Express, Trois-Rivières, 1968), 9.

70 P. Bouchard, La Nation, 24 novembre 1938. Le directeur ne semble pas se souvenir qu'il avait pourtant écrit le 8 octobre 1936: "Groulx nous a montré que la Confédération n'est pas viable. Nous en demandons la sécession dans l'avenir le plus immédiat (sic) et montrons ce qu'il faut construire." 
sible." ${ }^{71}$ On peut difficilement évaluer la part d'influence de l'abbé Groulx dans ce ralliement des séparatistes à l'autonomisme, puis au duplessisme. Le peu d'appuis qu'ils réussirent à susciter même à l'intérieur de la petite-bourgeoisie professionnelle, et la nouvelle conjoncture politique ne les incitaient pas à poursuivre seuls leurs débats intellectuels.

En conclusion, on peut affirmer que durant cette période l'abbé Groulx n'a jamais été séparatiste; d'ailleurs, ses prises de position officielles en faveur d'un "Etat français indépendant" ne furent qu'hypothétiques. Cependant comme il réfutait certaines thèses antiséparatistes des autonomistes, il apportait de l'eau au moulin des partisans du changement constitutionnel qu'il refusait de condamner. Il les considérait avec sympathie et était d'une certaine manière de connivence avec eux. D'ailleurs, au-delà des divergences sur les modalités politiques, il était spontanément du côté de ces radicaux petits-bourgeois intellectuels, qui préconisaient une "révolution nationale" et rejetaient la lutte des classes.

Nous ne sommes pas d'accord avec Jean-Pierre Gaboury lorsque dans son ouvrage sur Le Nationalisme de Lionel Groulx il déclare: "Il ressort de toute évidence de la pensée globale du maître nationaliste que son "Etat français" est plus qu'éventuellement, nécessairement un Etat indépendant. Ainsi la réputation de séparatiste qui l'accompagne est méritée." $72 \mathrm{Au}$ fond, en dissociant sa mystique nationale de l'action proprement politique, l'abbé Groulx maintenait le débat politique à un niveau si élevé qu'il était possible à chacun, autonomiste, fédéraliste ou séparatiste, d'y trouver sa justification et ses mots d'ordre partisans. Quant à l'abbé Groulx, s'il était prêt à se "résigner à l'inévitable" à cause du dépit que lui causait le fonctionnement de l'union fédérale, il n'a jamais cru que l'indépendance nationale était absolument nécessaire, pas plus qu'il n'a jamais préconisé l'indépendance du Québec comme solution immédiate.

Par-delà cette discussion, il est probablement plus important de rappeler que Groulx et les séparatistes partageaient la même illusion et entretenaient le même "mensonge bourgeois" 73 selon lequel l'Etat peut être libre et appelé à sauvegarder les intérêts de tous.

71 P. Bouchard, La Nation, 24 novembre 1938.

72 Jean-Pierre Gaboury, Le Nationalisme de Lionel Groulx (Ottawa, 1970), 156.

73 V. Lénine, "De l'Etat”, Oeuvres choisies, III : 320. 